\title{
Mechatronics at the University of Twente
}

\author{
Job van Amerongen and Rien Koster \\ Electrical Engineering Department and Cornelis J. Drebbel Research Institute for Systems Engineering, \\ University of Twente, P.O. Box 217, 7500 AE Enschede, Netherlands \\ e-mail: mailto:J.vanAmerongen@el.utwente.nl
}

\begin{abstract}
This paper describes some of the mechatronics activities at the University of Twente. In 1989, the founding of the Mechatronics Research Center Twente (MRCT) started a cooperation of the departments of Electrical Engineering, Mechanical Engineering, Applied Mathematics and Computer Science. The mechatronics activities get especially attention in projects in the Ph.D. program and in the 'Mechatronic Designer' program, but Msc. students participate as well. As an illustration of the philosophy behind the work at the University of Twente and of the activities carried out so far, the paper will describe two projects of the institute: the MART project and the ALASCA project.
\end{abstract}

\section{MART, a factory of the future}

The Mobile Autonomous Robot Twente (MART) project aimed in the first place at investigating how different disciplines can cooperate in a mechatronic team. The objective of such a team should be the development of a technical system with solutions

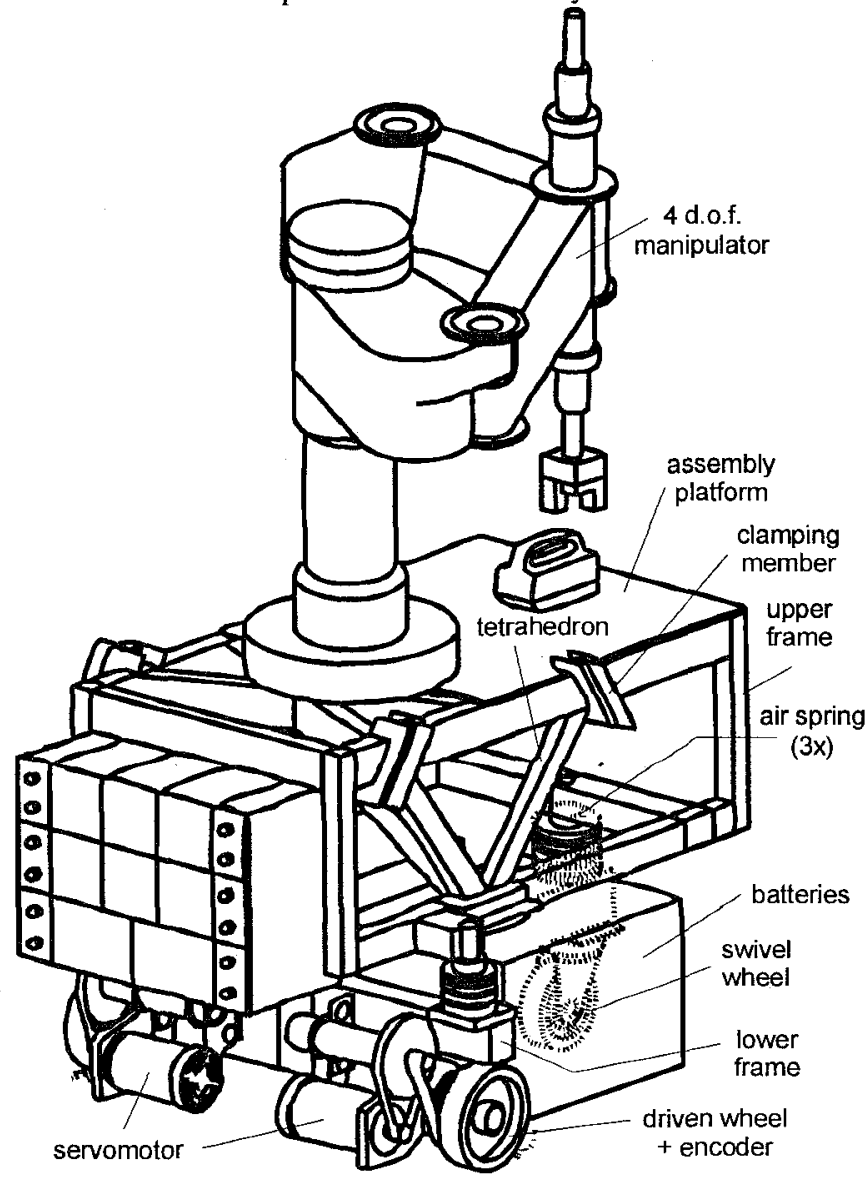

Fig. 1 The MART vehicle contributed from different disciplines. An automated assembly factory was adopted as a subject. It was a common effort of participants from mechanical engineering, control engineering and computer science. It resulted in an autonomously moving vehicle that, while riding a predestinated, product dependent route along a number of stocks, collects components and assembles them by a manipulator on board the vehicle. A vehicle, a manipulator, a gripper exchange system, a docking system, a navigation system together with all the hard- and software for task and path planning were developed, built together and tested.

Today consumer products are members of product families. Each member is assembled as a sub group of functional units. Functional units are produced and tested in large-scale factories. Final assembly takes place, close to the geographical marketplaces. Such an assembly factory will be a small-scale factory, able to quickly adapt to the demand in terms of types of products and quantities. MART is an example of such a factory [1]. It consists of stocks of functional units and of vehicles that move along a preset list of part supply stations, depending on the particular type of final product that is being manufactured. On board the vehicle, there is a 4-d.o.f. assembly robot (Fig.1). The robot takes components from a part supply system to the vehicle's deck and performs the assembly operations, even during riding. The design process started with the evaluation of basic concepts based on simple models. The outcomes of these evaluations directed the design of the different parts of the system. The more the designs grew, the more detailed the modeling became. It was interesting to see that deviations between the early predictions, the simulations in the final stage and the practical results, remained within $20 \%$. Consequently, simple modeling was of much use in order to direct the project.

\section{Vehicle}

One of the requirements the vehicle has to fulfil is offering the opportunity to the onboard manipulator to perform assembly while riding on an irregular floor. In the error budget of the tip (Fig. 2) with respect to the vehicle deck, $0.1 \mathrm{~mm}$ is available for positional error due to passing a threshold of $2.5 \mathrm{~mm}$. In order to achieve this, the frame was subdivided into a rigid lower frame with relatively rigid wheels, useful for odometry, and an upper frame, separated by relatively soft air springs. (Fig. 2) The upper frame contains the majority of the mass, especially the batteries. The optimal distribution of the weights was determined with simulations. Fig. 3 shows the error $\left(z_{\text {tip }}-z_{\text {vehicle }}\right)$ when passing the threshold at a speed of $1 \mathrm{~m} / \mathrm{s}$. The more mass is attributed to the upper frame the more it acts as a low pass filter. The upper frame will be the interface between the manipulator and the 
docking mechanism. The docking mechanism will ask for three points, rigidly connected to the manipulator base. Therefore, a tetrahedron-like upper frame was adopted (see Fig. 1). On the tip plane, the manipulator is carried. The lower frame (Fig. 1) is supported by a swivel wheel at the front and two servomotordriven wheels at the rear. These drives contain encoders as a provision for odometry.

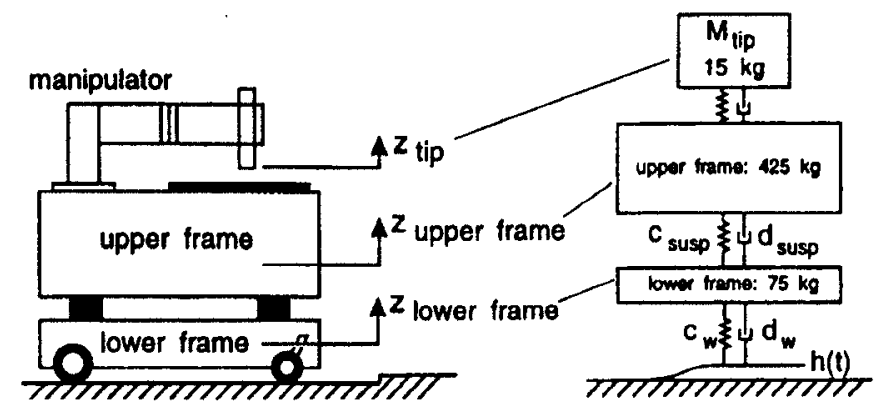

Fig. 2 Passing a threshold of $2.5 \mathrm{~mm}$

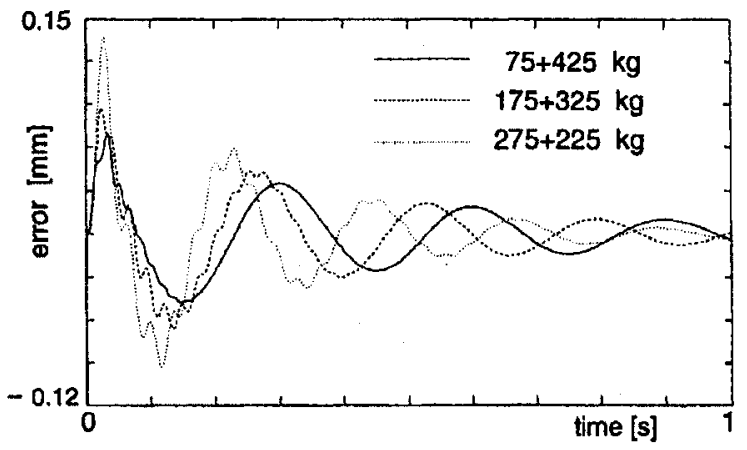

Fig.3 Errors when passing the threshold

\section{Tracking control}

The position $(x, y)$ and the orientation $(\varphi)$ of the vehicle are estimated by means of a Kalmanfilter that uses measurements from a beacon detection system as well as from odometry. As the vehicle is a system with a non-holonomic constraint no lateral corrections $\left(\Delta \dot{y}^{v}\right)$ can be made without moving in track direction (Fig. 4). Therefore, rather than taking as velocity-error signals:

$$
\begin{aligned}
& \Delta \dot{x}^{\nu}=\Delta v+\left[l-\cos \left(\Delta \varphi^{\nu}\right)\right] v_{r e f}+\omega \Delta y^{v} \\
& \Delta \dot{y}^{v}=\sin \left(\Delta \varphi^{v}\right) \nu_{r e f}-\omega \Delta x^{\nu} \\
& \Delta \dot{\varphi}^{\nu}=\Delta \omega
\end{aligned}
$$

a new orientation error signal has been defined:

$$
\Delta z^{v}=\Delta \varphi^{v}+\alpha \Delta y^{v} \operatorname{sign}\left(v_{r e f}\right)
$$

which replaces $\Delta \varphi$. This new orientation error is proportional to the lateral error (Fig. 4). The new concept of error measuring, called $x z$-error measure $[2,3]$ is expressed as:

$$
\begin{aligned}
& \Delta \dot{x}^{v}=\Delta v+\left[1-\cos \left(\Delta \omega^{v}\right)\right] v_{r e f}+\omega \Delta y^{v} \\
& \Delta \dot{z}^{v}=\Delta \omega+\alpha \sin \left(\Delta \varphi^{v}\right)\left|v_{r e f}\right|-\alpha \omega \Delta x^{v} \operatorname{sign}\left(v_{r e f}\right)
\end{aligned}
$$

The parameter $\alpha$ determines the strength of steering towards the reference track.

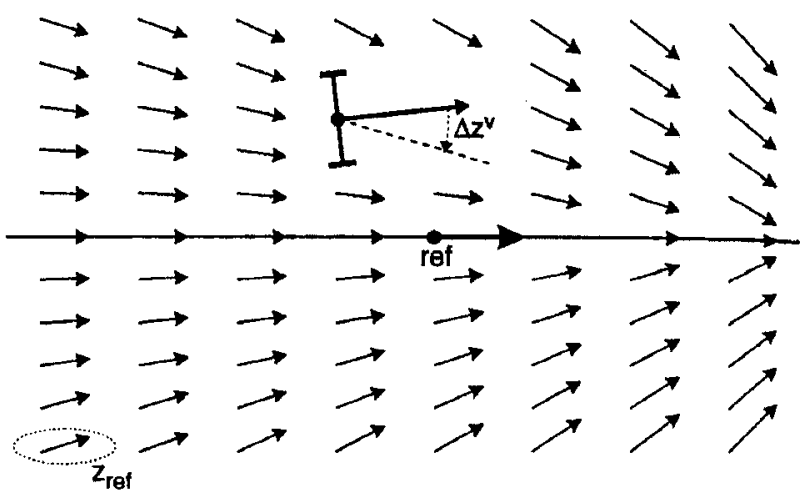

Fig. 4 Strength of steering towards the reference track

A simple steering law is applied:

$\Delta \dot{x}_{v}=-K_{x} \Delta x^{v} ; \Delta \dot{z}^{v}=-K_{2} \Delta z^{v}$

Let $\Delta v=\delta v_{r e f}$ and $\Delta \omega=\delta \omega_{\text {ref }}$ be the correction signals needed to keep the vehicle on track:

$$
\begin{aligned}
& \delta v_{r e f}=-K v_{x} \Delta x^{v}-\left(1-\cos \left(\Delta \varphi^{v}\right)\right) \nu_{r e f}-\omega \Delta y^{v} \\
& \delta \omega_{r e f}=-K_{z} \Delta z^{v}-\alpha \sin \left(\Delta \varphi^{v}\right)\left|v_{r e f}\right|-\alpha \omega \Delta x^{v} \operatorname{sign}\left(v_{r e f}\right)
\end{aligned}
$$

The vehicle's dynamics are, with reference to Fig. 5:

$$
\begin{aligned}
& M \dot{v}=h M \omega^{2}+F_{l}+F_{r}-F_{f l}-F_{f r} \\
& \left(J+M h^{2}\right) \dot{\omega}=h M v \omega+b\left(F_{r}-F_{l}-F_{f r}+\right. \\
& F_{f l}=K_{l} \operatorname{sign}\left(v_{\omega l}\right) ; F_{f r}=K_{r} \operatorname{sign}\left(v_{\omega r}\right) \\
& F_{\mathrm{fl}} \rightarrow F_{l} \\
& F_{\mathrm{fr}}
\end{aligned}
$$

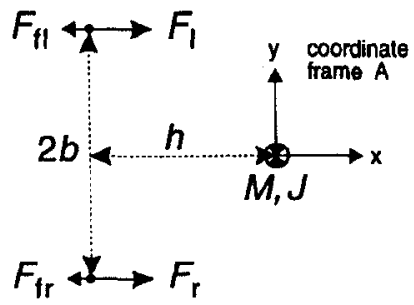$$
\left(J+M h^{2}\right) \dot{\omega}=h M v \omega+b\left(F_{r}-F_{l}-F_{f r}+F_{f l}\right)
$$

The nonlinearities, $h M \omega^{2}$ and $h M v \omega$ are compensated in a feedback loop. Consequently, when the vehicle moves along a reference trajectory (Fig. 6) of $12 \mathrm{~m}$ length (in $34 \mathrm{~s}$ ), the absolute value of the positional error never exceeds $10 \mathrm{~mm}$. Similar results could be obtained with a learning feedforward controller that only requires a very simple model of the vehicle [6].

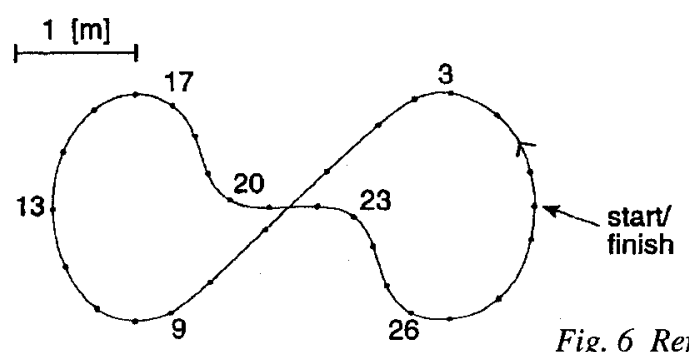

Fig. 6 Reference track 


\section{Product Supply Station}

The Product Supply Station (PS) contains a stock, a stock unloading system and a docking mechanism. The vehicle approaches the docking mechanism with the accuracy of the tracking system (error less than $10 \mathrm{~mm}$ ). The docking mechanism simply clamps the vehicle in such a way that no degree of freedom is left. Three leaf springs, each fixing two independent coordinates onboard the vehicle, are kept by a clamp, based on the PS. These leaf springs, when fitted to the corners of the tetrahedron (Fig. 1), enable an extremely rigid connection between the manipulator base and the PS as long as the vehicle remains docked. The manipulator moves overhead the PS, recognizes a set of markers and so relates the vehicle frame to the PS-frame. This solution depends on robot vision and coordinate transformation rather than on interrelating the two frames in a pure mechanical way.

\section{Manipulator}

The manipulator (Fig. 7) consists of two sets, each containing a drive $(9,8,7,5)$ a driving shaft (4), a double upper arm (3) and a double lower arm (2).

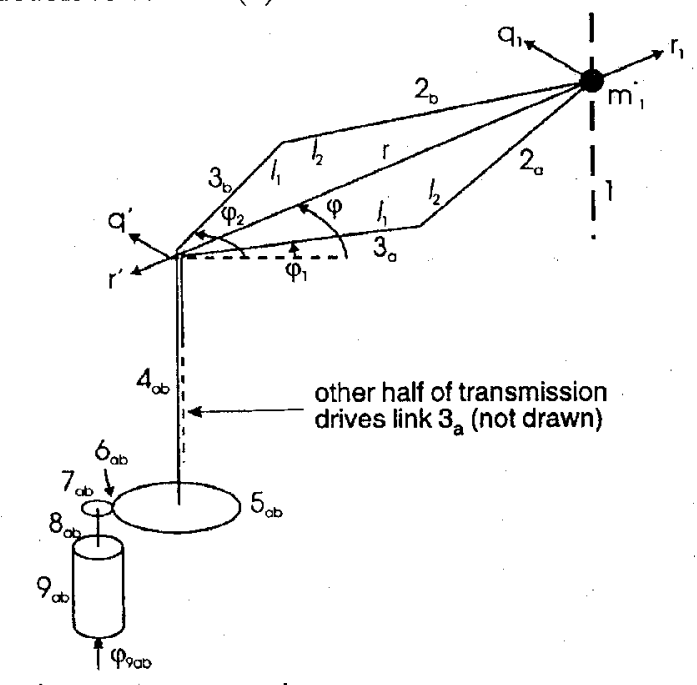

one half drawn: drives link $3_{\mathrm{b}}$

Fig. 7 The manipulator

Both lower arms handshake at the end effector (1). This two d.o.f. $(r, \varphi)$ configuration features two drives in the pedestal, with a minimum of moving mass. The arms have been designed as thin-walled box-like structures that combine high stiffness and low mass. With the servomotors (9) blocked, lowest natural frequencies of the mechanics turn out to be between $60 \mathrm{~Hz}$ and 130 Hz. The end effector itself has two d.o.f.: rotation around its axis (1) and travel along that axis. Both servomotors for these d.o.f. are in the pedestal as well. In order to achieve sufficient bandwidth, a highly stiff transmission between these motors and the end effector was arranged. It consists of two shafts, concentric with the hollow shafts (4) with timing belts through the hollow arms. Because of the relatively high natural frequencies of the manipulator a $\mathrm{PD}$-controller for each motor was sufficient to meet the requirements.

The gear wheels (7) and (5) act under controlled preloading in order to avoid backlash on one hand and to minimize hysteresis because of friction on the other hand [7]. The gear preload by pressing the motor shaft pinion against the gear wheel is generated by an electromagnet (Fig. 8). This gear preload should, in principle, be equal to

$F_{y}=\frac{(T-J \ddot{\varphi})(\tan \alpha)}{r}=\frac{\left(K_{\mathrm{m}} I-J \ddot{\varphi}\right)(\tan \alpha)}{r}$

where $T$ is the electromagnetic motor torque, $J$ is the mass moment of motor inertia, $r$ the radius of the pinion, $\alpha$ the constant gear geometry pressure angle and $K_{\mathrm{m}}$ the motor constant. A slightly more extended algorithm has been implemented according to Fig. 8 . The acceleration $(\ddot{\varphi})$ and the velocity $(\dot{\varphi})$ are estimated by an observer. The analog root extractor compensates for the nonlinearities in the magnet actuator

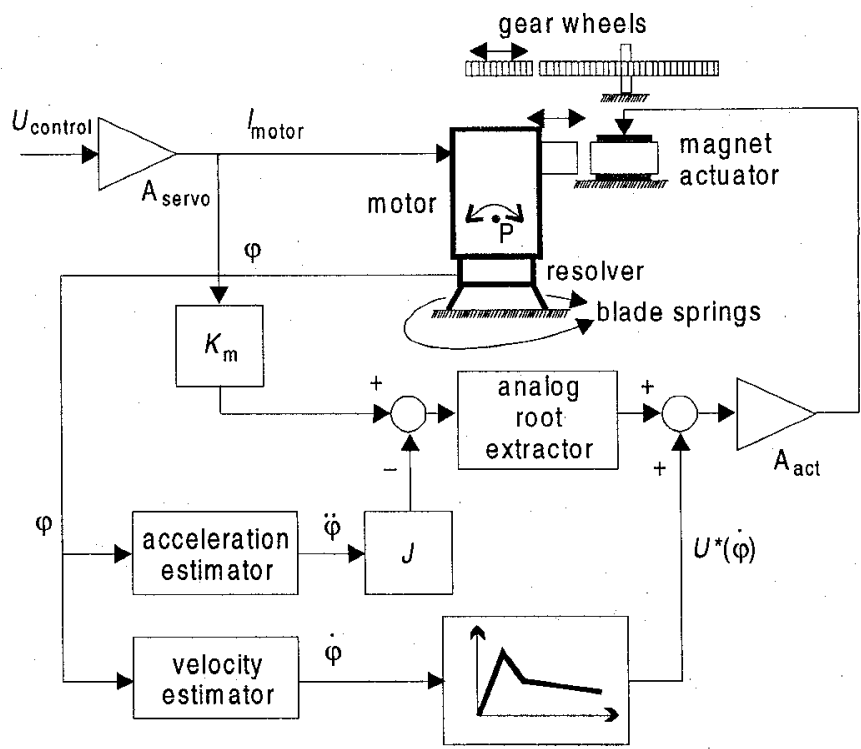

Fig. 8 System for controlled gear preload

\section{Navigation}

Three unique active infrared beacons are sufficient to determine the vehicle's pose according to Snellius' method of triangulation (Fig. 9) [4].

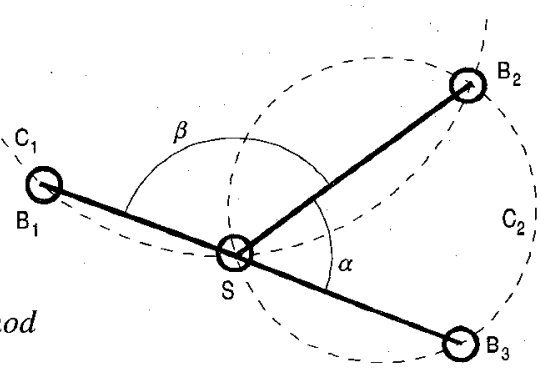

More than three beacons may be available. A rotating $(4 \mathrm{~Hz})$ infrared sensor detects the sequential beacons. The model of the sensor is expressed as

$\alpha_{n}=a \tan \frac{b_{y, i}-y_{n}}{b_{x, i}-x_{n}}-\varphi_{n}+v_{n}$ 
where $\left(x_{n}, y_{n}, \varphi_{n}\right)$ is the vehicle pose (axis of the rotary detector) and $\left(b_{x i}, b_{y i}\right)$ is the position of the beacon $i$. Furthermore $\alpha_{n}$ is the measured angle and $v_{n}$ is the measurement noise. Since the vehicle is moving, the time instant corresponding to the measured $\alpha_{n}$ is saved as well. The beacon detection system with a scanning frequency of only $4 \mathrm{~Hz}$ is cooperating with an odometry system. Each of the driving wheels is equipped with an angular encoder. If perfect rolling exists the curvilinear distance is

$l=\frac{r\left(\varphi_{\omega 1}+\varphi_{\omega 2}\right)}{2}, \varphi=\frac{r\left(\varphi_{\omega 2}-\varphi_{\omega 1}\right)}{b}, b$ is the wheel base.

The change of the vehicle's coordinates is approximated by

$$
\Delta x=\Delta l\left(\cos \varphi+\frac{\Delta \varphi}{2} \sin \varphi\right), \quad \Delta y=\Delta l\left(\sin \varphi-\frac{\Delta \varphi}{2} \cos \varphi\right)
$$

where $\Delta \varphi=\varphi(t)-\varphi(t-\Delta t)$.

The beacon detection system and the encoder system are brought together in a discrete Kalman filter structure [4] in such a way that the beacon measurements re-adjust the pose estimate from the odometry. The vehicle model in the Kalman filter was kept as simple as possible; only the servomotor dynamics and the vehicle dynamics were modeled. This combination of two navigation systems combines the advantages of both: no drift and a high pose sampling rate allowing a tracking bandwidth much higher than $4 \mathrm{~Hz}$.

\section{Computer hard-and software}

The hardware consists of onboard transputer hardware, a radio link and a ground based computer. The onboard computing power performs all the control for driving, manipulating, pose detection, the gripper exchange system and the robot video cameras. All the processing related to task of the factory such as product information and routing is performed by the ground based computer. The transputer hardware enabled the design of a modular software system.

\section{Conclusions}

Useful combination of mechanics, control and software made the system work according to the preset requirements. Collaboration between different disciplines is a critical factor for success in mechatronic projects. Modeling is a medium of mutual understanding. This project was completed within 5 years. The project team consisted of a senior project leader and four project leaders, one for each discipline. Close cooperation existed with staff of the mechanical and the electrical workshops. About 50 students completed their MSc.--thesis within this project.

\section{Alasca Project}

The Alasca project (Automated Laser Aided Servo Controlled Assembly) is an example of a project in the Mechatronic Designer program. This is a two-year program for students with a Master's degree in engineering (mainly EE and ME). During one year, students from e.g. electrical engineering follow additional courses in mechanical engineering and advanced courses in other subjects. In the second year, they have to design a mechatronic system, preferably a real industrial system, under the guidance of experienced designers. The Alasca project was done in cooperation with Philips. It resulted in the design of a fast manipulator for placing Surface Mount Devices (SMD's) on a printed circuit board (PCB), combining a linear and rotational electrical servo [5]. Two students (one from mechanical and one from electrical engineering) where involved in the manipulator design. A third student did a complimentary project, resulting in a system for laser welding of the leads of the SMD, as a replacement of the presently used soldering techniques.

\section{Design objective}

The main objective of the design was the replacement of the presently used pneumatic manipulator by a well controllable electrical servo system, able to perform translational and rotational motions, while placing an SMD on a PCB. Compared with the preumatic devices the new device should have a higher position accuracy and should be able to control:

- impact velocity (and force)

- press on force

- orientation of each manipulator separately

- z-position, allowing limitation of the applied stroke.

The final design had to meet the following specifications:

\begin{tabular}{|c|c|c|c|}
\hline max. diameter & $80 \mathrm{~mm}$ & Total mass & $<3 \mathrm{~kg}$ \\
\hline z-motor & & $\varphi$-motor & \\
\hline stroke & $70 \mathrm{~mm}$ & range & $\geq 2 \pi \mathrm{rad}$ \\
\hline $\begin{array}{l}\text { accuracy } z \text {-direction } \\
\text { accuracy } x, y \text {-direction }\end{array}$ & $\begin{array}{l} \pm 10 \mu \mathrm{m} \\
\pm 5 \mu \mathrm{m}\end{array}$ & $\begin{array}{l}\text { accuracy } \\
\text { controlled band- } \\
\text { width }\end{array}$ & $\begin{array}{c} \pm 0.35 \mathrm{mrad} \\
100 \mathrm{~Hz}\end{array}$ \\
\hline controlled bandwidth & $100 \mathrm{~Hz}$ & max. acceleration & $1200 \mathrm{rad} \mathrm{s}^{-2}$ \\
\hline max. force & $10 N$ & & \\
\hline max. acceleration & $100 \mathrm{~ms}^{-2}$ & & \\
\hline
\end{tabular}

In addition, the manipulator should have:

- a vacuum nozzle at the end of the translator

- long lifetime

- little maintenance

- independent operation of $\varphi$ - and $z$-motions.

The primary choice to be made was the type of motor. DC-motors have the advantage that they can easily be controlled. AC induction motors on the other hand can have a light and robust construction, but require more complex control electronics.

\section{Design}

A high accuracy and bandwidth require that the moving parts are as light as possible and that friction is minimized. It was decided to develop an induction motor able to rotate and translate simultaneously. The basis for the design is a tubular motor, consisting of a number of concentric cylinders. Starting at the outside, the motor consists of:

- stator with the motor coils

- translator/rotor tube (a hollow aluminum tube)

- back iron.

Due to the magnetic fields in the motor coils, currents are induced in the 'translator' tube. The back iron is needed in order to 
obtain a high magnetic flux. The only moving part is the lightweight, hollow 'translator'. This tube is supported by means of two air bearings that give the system a high stiffness and eliminate friction. Because it is hollow, the vacuum for the nozzle can be applied through this tube. All parts of the motor can be made from easily available standard tubes. Two sets of coils enable the 'translator' to translate and rotate simultaneously. The coil configuration is given in Fig. 10. TRIM stands for Tubular Rotational Induction Motor and TLIM for Tubular Linear Induction Motor.

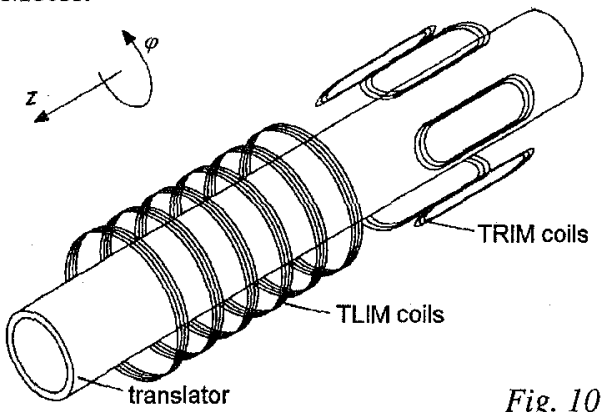

Fig. 10 Coil configuration

Together with appropriate power electronics, these induction motors should behave as DC-motors. A new patented linearizing feedback was designed that combined minimum dissipation with good control properties. The main advantage is that when no force or torque is required, the motor current is zero.

\section{Sensors}

To maintain the nice properties of the contactless translator, contactless sensors are required as well. As a proper sensor for the $z$-motion was not commercially available, it was developed in the project. The sensor consists of a single coil in which a magnetic core is moved. The non-linearity of the sensor is compensated by means of a look-up table, but it introduces a position dependent resolution. Special care was taken to prevent disturbing fields of the motor from influencing the sensor. The design of a contactless rotational sensor is more complex, because of the translations. No commercially available solutions were found. A new patented sensor was developed according to Fig. 11. Focussed light from (in principle) three LED's is projected at three photo diodes. LED's and photo diodes are mounted at the stator. A sheet of polarized material connected to the translator tube changes the polarization of the light sources when the translator rotates. A small sheet of polarized material is placed in front of each photo diode as well. The directions of polarization of the latter differ exactly $120^{\circ}$. This enables the angle to be measured. In order to improve the accuracy and reduce the sensitivity for noise, the applied light is sinusoidally modulated. By means of a standard resolver-to-digital converter, the signals from the photo diodes can be directly converted into a digital signal with 14 bits of resolution. Therefore, the sensor has been called an optical resolver.

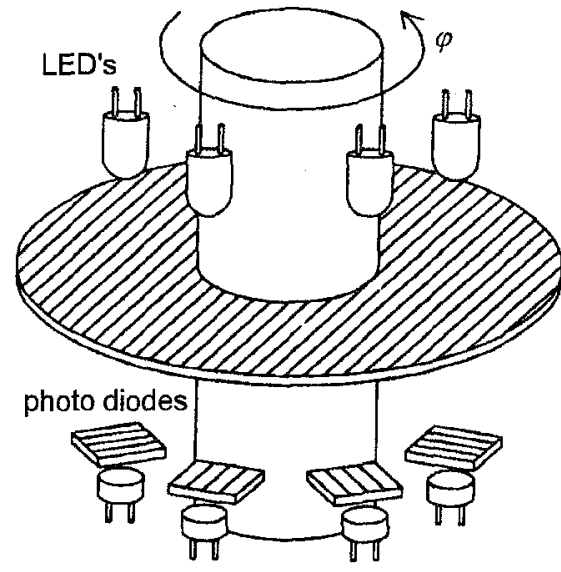

Fig. 11

Optical resolver

\section{Results}

The result of the project was a prototype of the manipulator that met most of the design specifications. Because there is no contact at all between the 'translator' and the outside world, it can rotate over an arbitrary angle. The maximum force as well as the maximum moment was larger than requested. The resolution of the sensors was sufficient. The attained accuracy was $25 \mu \mathrm{m}$ for the translation and $0.09 \mathrm{mrad}$ for the rotation. The translational accuracy was less than specified but seems to be good enough for the tasks to be performed. In the experiments, the bandwidths of the controlled system were limited to $40 \mathrm{~Hz}$ for the rotational motion and $50 \mathrm{~Hz}$ for the translational motion. By using a second order path generator in the controller a linear motion of $70 \mathrm{~mm}$ could be made in $50 \mathrm{~ms}$, without overshoot.

\section{References}

[1] J. Abrahams (1990), Philips Electronics NV Eindhoven The Netherlands, Private communication

[2] W. Oelen, Modelling as a tool for design of mechatronic systems, $\mathrm{PhD}$.--Thesis, University of Twente, 1995

[3] W. Oelen and J. van Amerongen, Robust Tracking Control of Two Degrees of Freedom Mobile Robots, Control Engineering Practice, Vol. 2, No. 2, April 1994, pp. 333-340, 1994

[4] A.J. de Graaf, On-line measuring systems for a mobile vehicle and a manipulator gripper, $\mathrm{PhD}$.-Thesis, University of Twente, 1994

[5] T.G. Blömers and P.T. Rutgers, Design and implementation of $a z-\varphi$ controlled pipette, Report mechatronic design course, Control Laboratory, Dept. of EE, University of Twente, 1993

[6] Starrenburg, J.G., W.T.C. van Luenen, W. Oelen and J. van Amerongen, Learning Feedforward Controller for a Mobile Robot Vehicle, Control Engineering Practice, Vol. 4, No. 9, pp. 1221-1230

[7] Kruijer, C.W., Geregeld voorspannen van tandwielen, MSc thesis, University of Twente, 1992 (in Dutch) 